\title{
IDENTIFICATION OF THE ORB WEAVING SPIDER (ARANEAE: ARANEIDAE) FAUNA OF DIR LOWER (PAKISTAN) THROUGH DNA BARCODING
}

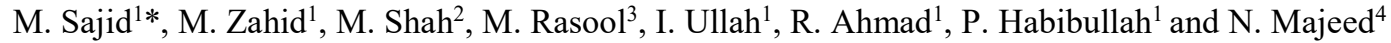 \\ ${ }^{1}$ Department of Zoology Islamia College University, Peshawar, Pakistan; ${ }^{2}$ Department of Zoology Government Superior \\ Science College Peshawar; ${ }^{3}$ Government Degree College Kabal Swat, Pakistan; ${ }^{4}$ Department of Zoology, University of \\ Punjab, Lahore-Pakistan \\ *Corresponding author's email: muhammadsajid541@gmail.com
}

\begin{abstract}
As a result of faunistic study on the Orb weaving spider fauna of Pakistan one genus, Gibbaranea Archer, 1951 and six species, Argiope versicolor (Doleschall, 1859), Argiope lobata (Pallas, 1772), Neoscona polyspinipes (Yin, Wang, Xie and Peng, 1990), Neoscona scylla (Karsch, 1879), Gea spinipes (C. L. Koch, 1843) and Gibbaranea ullrichi (Hahn, 1835) are recorded from Pakistan for the first time and the presence of five species are confirmed. Eriovixia excelsa (Simon, 1889) and Araneus mitificus (Simon, 1886) are recorded for the first time since 1935.COI sequences $>200$ bp recovered from 23 specimens were analyzed using neighbor-joining trees and Barcode Index Numbers (BINs)Nucleotide alignment for each species is provided with Sample id, Process id, match process id and match BIN. A map showing the local distribution of each species is provided.
\end{abstract}

Key words: Dir Lower, Aranei, new records, distribution, taxonomy.

\section{INTRODUCTION}

The family Araneidae Clerck (1757) consists of 178 valid genera and 3051 species (WSC, 2020). Distribution of araneids is cosmopolitan. They are mostly found in damp places and rarely in arid places. Nearly all the species form orb web to live in. They construct web in herbs, shrubs, foliage, grasses and in trees. If disturbed, they fall down and use the same thread for climbing again. Generally smaller size is observed in the males. Males are rarely seen in the web as compared to females (Gajbe, 2005). Members of the family are reported poor and a relatively small number of new species has been described from Pakistan (Mukhtar, 2012). Simon (1889, 1907, 1918), Caporiacco (1934), Dyal (1935) described new species of the family from the country. Arshad et al. (1984), Khatoon (1986) recorded some species of the genus Neoscona from Pakistan and placed them in genus Araneus which were later on transferred to genus Neoscona. Tikader (1982), Qadir (1997), Ghafoor (2002), Ghafoor and Beg (2002), Parveen (2003), Butt and Siraj (2006), Mukhtar and Mushtaq(2005), Mukhtar (2012), Mukhtar and Tahir (2013), attempted to provide taxonomic description of Pakistani Araneid. Razzaq (2002) an unpublished data and Kazim et al. (2015) reported the family from Northern Areas of Pakistan.

Morphological identification of spiders has many drawbacks i.e. a time consuming and challenging task due to polymorphism, sexual dimorphism and lack of keys to identify juveniles. To consider this obstacle and to clarify all the ambiguities, the use of additional tools for rapid species identification is indispensable (Tyagi et al., 2019). DNA barcode provides an alternate approach to identify specimens (Ashfaq et al., 2019). For accurate species identification, to resolve taxonomic questions, population estimation, detection of cryptic species and species complexes, DNA barcode has been widely used in the last decade in biodiversity research (Tyagi et al., 2019). BOLD greatly facilitate the use DNA barcode for species identification. It enables species discrimination by assigning COI sequence clustered to BIN (Ashfaq et al., 2019).

Present study was carried out with the aim to find out the diversity of family Araneidae at Dir Lower Pakistan. Phylogenetic alignment was carried out for the $1^{\text {st }}$ time for new data of the family Araneidae from the country.

\section{MATERIALS AND METHODS}

Collection was made from 2017 to 2019. Different localities and habitats were searched out for the collection process at District Dir Lower Pakistan. Spiders were hand collected and preserved in $80 \%$ ethanol for morphological study and in $95 \%$ ethanol for molecular work. Specimens were examined under a LABOMED, INC. Los Angeles, USA (LB-344) stereomicroscope with $2 \mathrm{x}$ and $4 \mathrm{x}$ zoom lens. Nomenclature follows the World Spider Catalog (2020). The studied specimens are kept in Museum of Zoology Department Islamia College University Peshawar. Some specimens were sent for DNA barcoding to the Centre for Biodiversity Genomics, 
Biodiversity Institute of Ontario, University of Guelph, Canada. DNA extraction, PCR and sequencing were performed with using Standard protocol (http://ccdb.ca/resources/) (Ashfaq et al., 2019). Nucleotide sequence alignments of COI-5P are generated by using Codon Code aligner version 8.02.

Abbreviations: MICU- Museum Islamia College University Peshawar; m-meter; WSC- World Spider Catalog; ICUP- Islamia College University Peshawar: SPKPK- Spider Project Khyber Pakhtunkhwa; BINbarcode index number.

\section{RESULTS}

Argiope lobata (Pallas, 1772)

Fig 2, Map

Aranea lobata Pallas, 1772: 46, figs 14-15 (D + ).
Argiope lobata Thorell, 1873: 520; Argiope lobata Jäger, 2012c: 288, figs 44-48 (ぷ+ ).

Sample id: ICUP-MS-0052

Process id: SPKPK052-20

Match BIN: BOLD: AAZ8817

Material Examined: PAKISTAN: 1 q (MICU, MP-170), Banda Hills, Talash, Dir Lower, Khyber Pakhtunkhwa $\left(34.77111^{\circ} \mathrm{N}, 71.95761^{\circ} \mathrm{E}\right), 1374 \mathrm{~m}$ a.s.1., 05.07.2018,

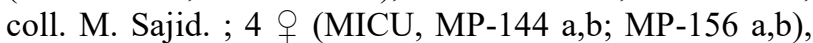
Zombaqy Hills, Talash, Dir Lower, Khyber Pakhtunkhwa $\left(34.73737^{\circ} \mathrm{N}, 71.94780^{\circ} \mathrm{E}\right), 1012 \mathrm{~m}$ a.s.1., 03.07.2018, coll. M. Sajid.

Distribution: This species is distributed from Southern Europe to Central Asia and China, Northern Africa, South Africa, Israel, India, from Myanmar to New Caledonia and Northern Australia (WSC, 2020). The species is recorded for the first time from Pakistan.

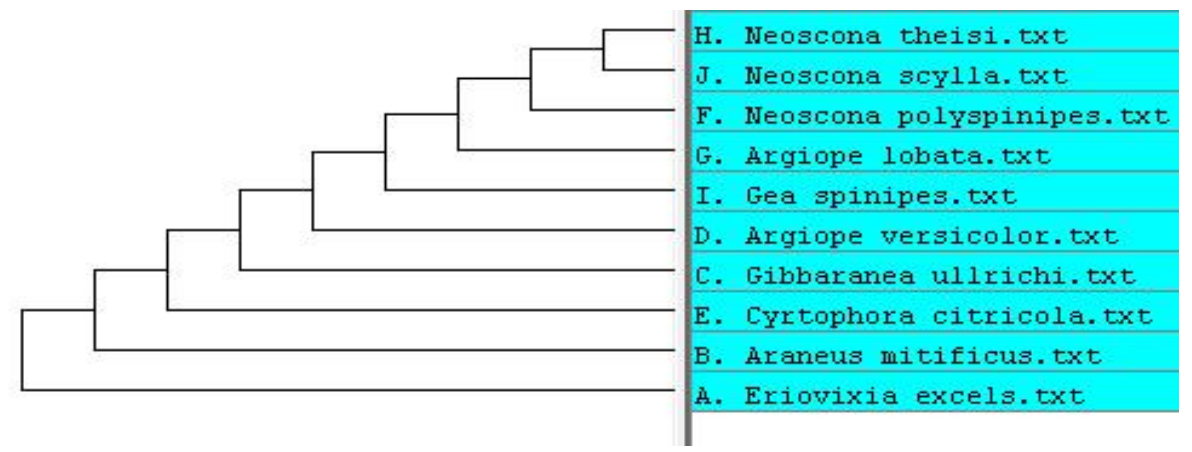

Figure 2: Homology Dendogram showing genetic similarity among Eriovixia excelsa (Simon, 1889), Araneus mitificus (Simon, 1886) Gibbaranea ullrichi (Hahn, 1835), Argiope versicolor (Doleschall, 1859), Cyrtophora citricola (Forsskal, 1775), Neoscona polyspinipes (Yin, Wang, Xie\& Peng, 1990), Argiope lobata (Pallas, 1772), Neoscona theisi (Walckenaer, 1841), Gea spinipes (C. L. Koch, 1843) and Neoscona scylla (Karsch,

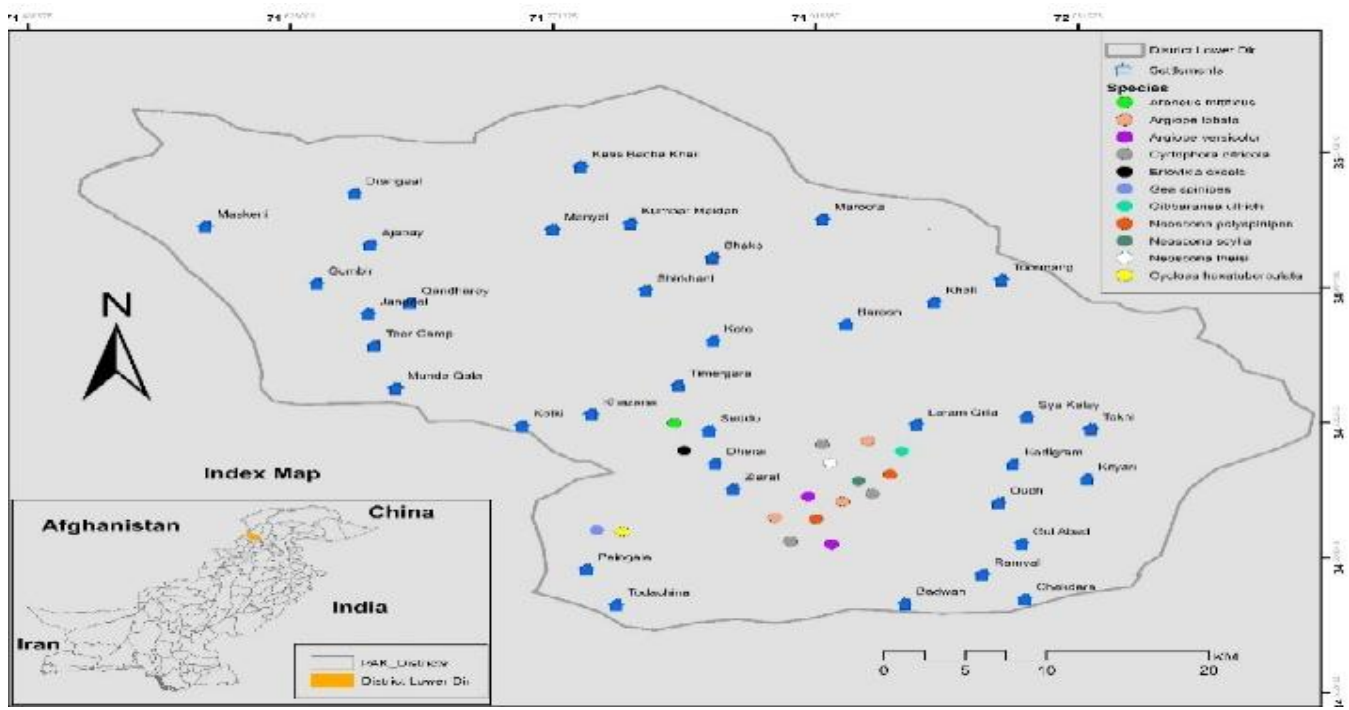

1879).

Map. Araneidae records from District Dir Lower, Khyber Pakhtunkhwa, Pakistan.

Argiope versicolor (Doleschall, 1859)
Fig 1, Map

Epeira versicolor Doleschall, 1859: 31, figs 10 (D $)$ ). 
Argiope versicolor Jäger \& Praxaysombath, 2009: 38, figs 69-73 (ぷ+).

Sample id: ICUP-MS-0040 and ICUP-MS-0045

Process id: SPKPK040-20 and SPKPK045-20

Match BIN: BOLD: ACG0576

Material Examined: PAKISTAN: 1 (MICU, MP-08), Barcharay Hills, Talash, Dir Lower, Khyber Pakhtunkhwa $\left(34.71647^{\circ} \mathrm{N}, 71.91671^{\circ} \mathrm{E}\right), 1268 \mathrm{~m}$ a.s.1., 10.05.2018; 1 ㅇ
(MICU, MP-82), Barcharay (Jarjoray Hills), Talash, Dir Lower, Khyber Pakhtunkhwa $\left(34.71667^{\circ} \mathrm{N}, 71.91701^{\circ} \mathrm{E}\right)$, 1310 m a.s.1., 18.06.2019, all. coll. M. Sajid.

Distribution. The species is distributed from China to Indonesia (WSC, 2020). Recently recorded from India (Tyagi et al., 2019). Present study confirms its existence in Pakistan (Northern areas) for the first time.

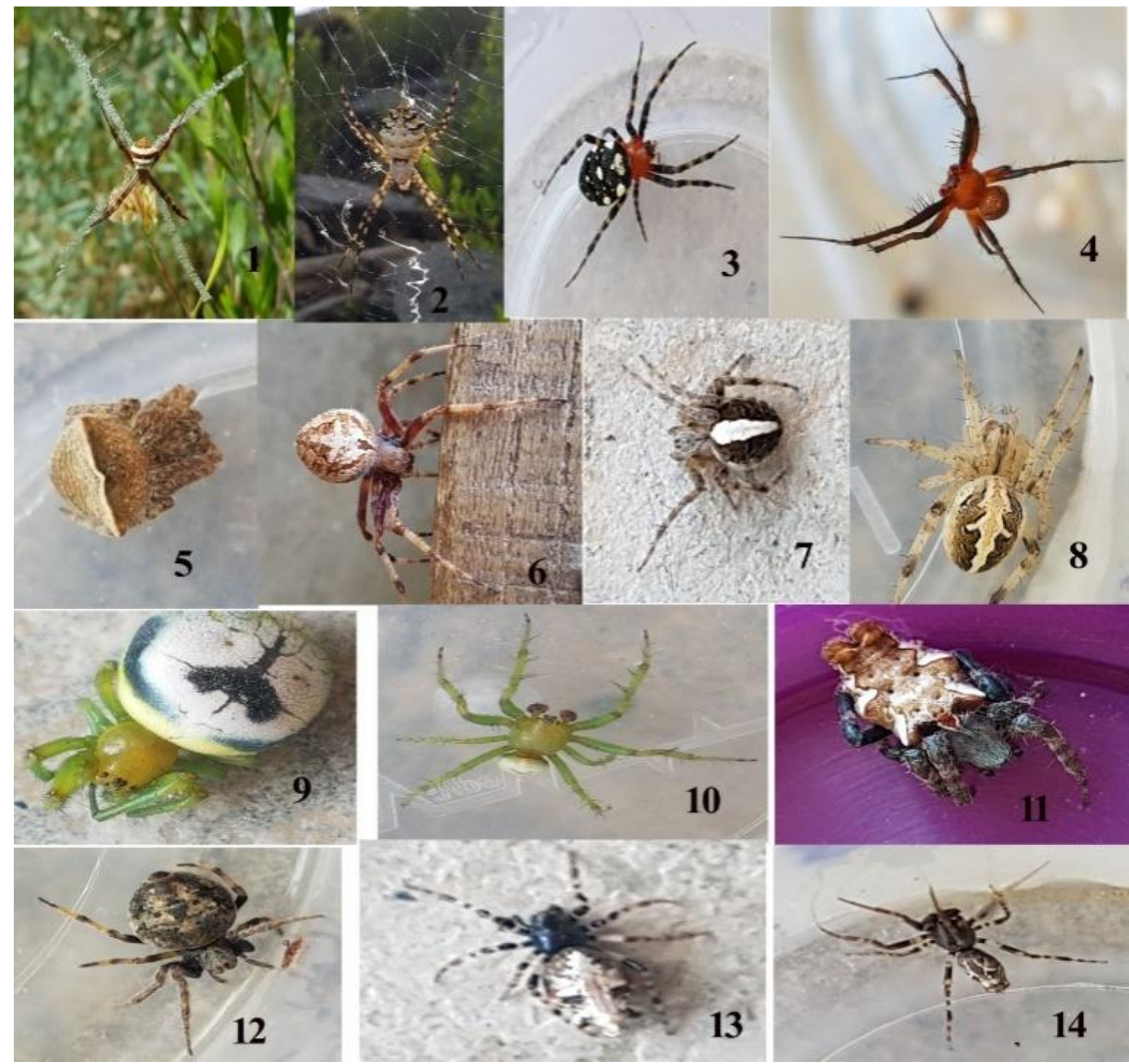

Figs 1-14. 1-Argiope versicolor; 2-Argiope lobata; 3-female Gea spinipes; 4-Male Gea spinipes; 5-Gibbaranea ullrichi; 6-Neoscona polyspinipes; 7-Neoscona Scylla; 8-Neoscona theisi; 9-female Araneus mitificus; 10_-male Araneus mitificus; 11-Cyrtophora citricola; 12_Eriovixia excels; 13-14_female \& male Cyclosa hexatuberculata.

1-70 Nucleotide sequence alignment 

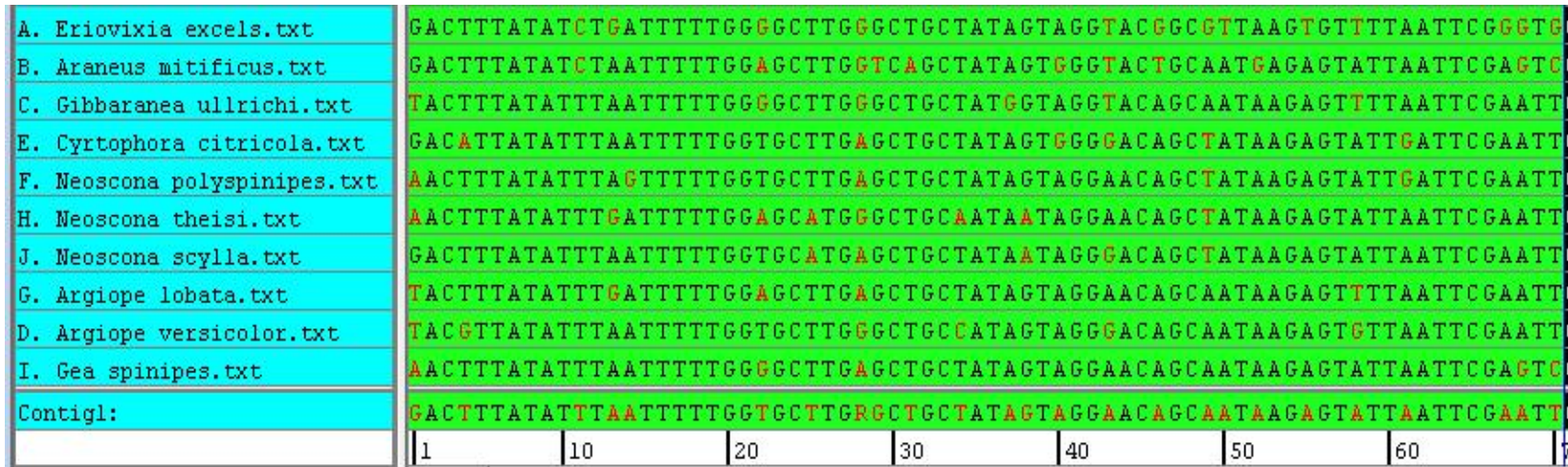

71-140 Nucleotide sequence alignment

\begin{tabular}{|l|}
\hline A. Eriovixia excels.txt \\
\hline B. Araneus mitificus.txt \\
C. Gibbaranea ullrichi.txt \\
E. Cyrtophora citricola.txt \\
F. Neoscona polyspinipes.txt \\
H. Neoscona theisi.txt \\
J. Neoscona scylla.txt \\
G. Argiope lobata.txt \\
\hline D. Argiope versicolor.txt \\
\hline I. Gea spinipes.txt \\
\hline Contigl: \\
\hline
\end{tabular}

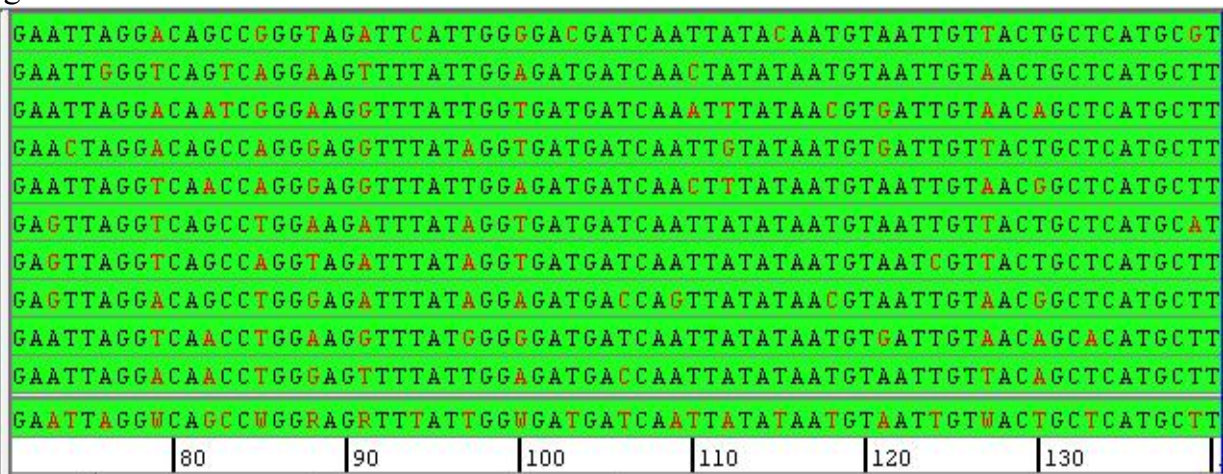

141-210 Nucleotide sequence alignment

\begin{tabular}{|l|}
\hline A. Eriovixia excels.txt \\
B. Araneus mitificus.txt \\
\hline C. Gibbaranea ullrichi.txt \\
E. Cyrtophora citricola.txt \\
F. Neoscona polyspinipes.txt \\
H. Neoscona theisi.txt \\
J. Neoscona scylla.txt \\
G. Argiope lobata.txt \\
D. Argiope versicolor.txt \\
I. Gea spinipes.txt \\
\hline Contigl: \\
\hline
\end{tabular}

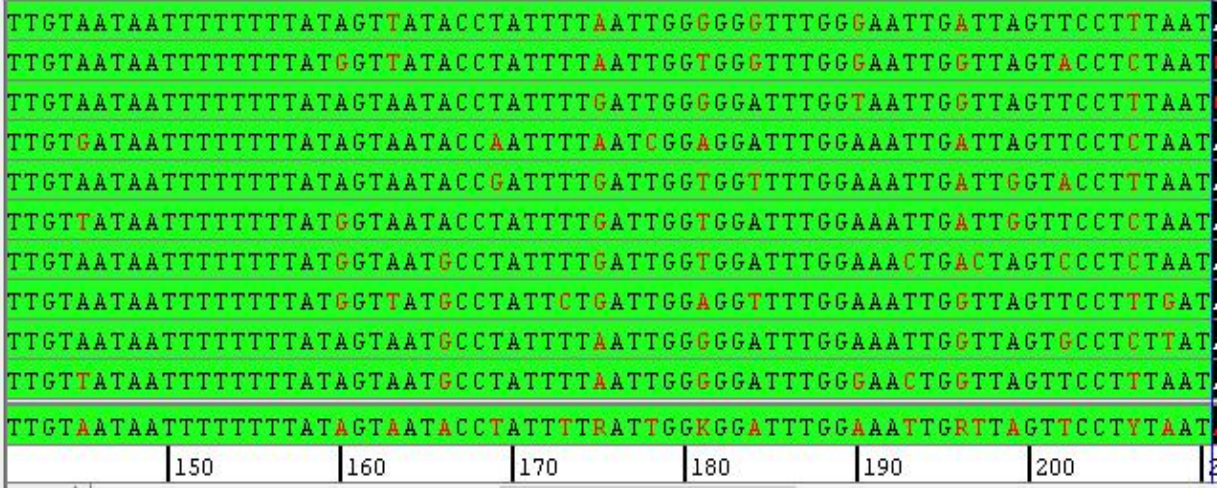

211-280 Nucleotide sequence alignment

\begin{tabular}{l|} 
A. Eriovixia excels.txt \\
B. Araneus mitificus.txt \\
C. Gibbaranea ullrichi.txt \\
E. Cyrtophora citricola.txt \\
F. Neoscona polyspinipes.txt \\
H. Neoscona theisi.txt \\
J. Neoscona scylla.txt \\
G. Argiope lobata.txt \\
D. Argiope versicolor.txt \\
I. Gea spinipes.txt \\
\hline Contigl: \\
\hline
\end{tabular}

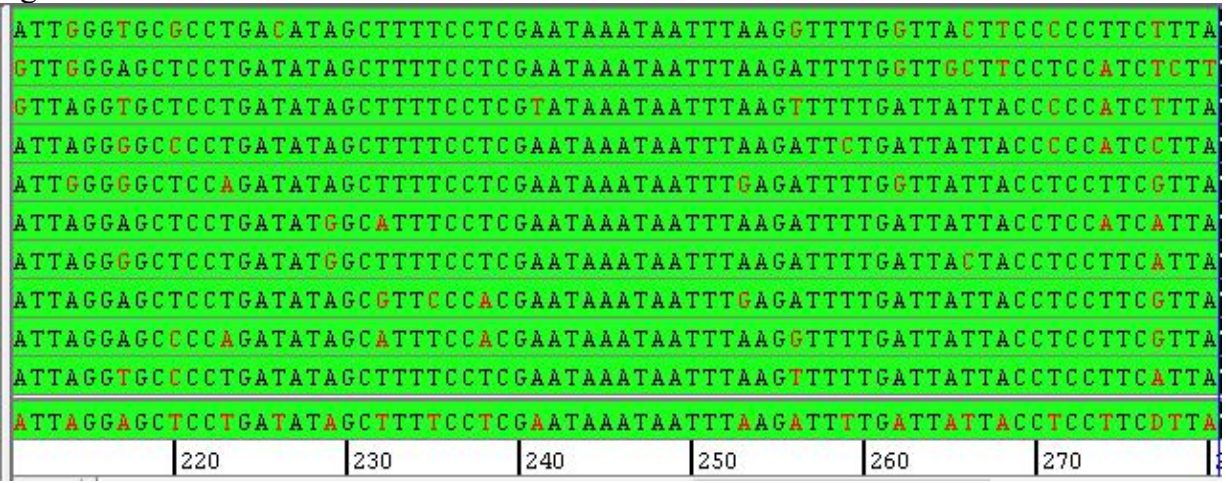

281-350 Nucleotide sequence alignment 


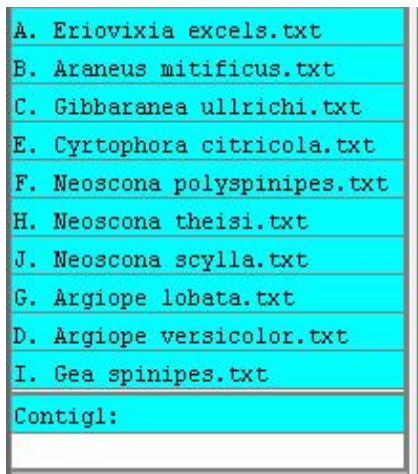

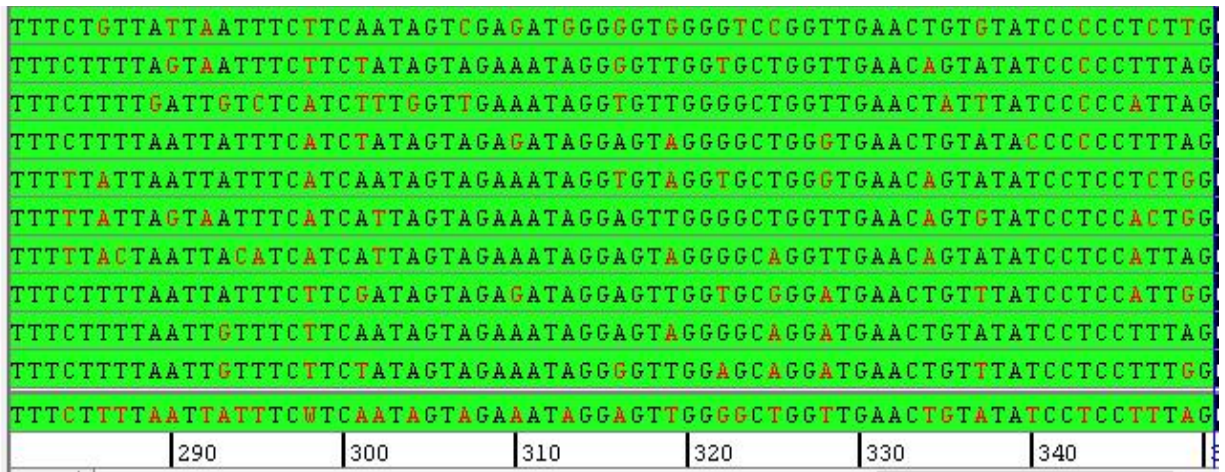

351-420 Nucleotide sequence alignment

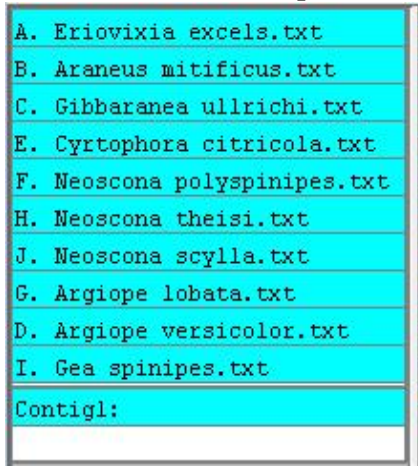

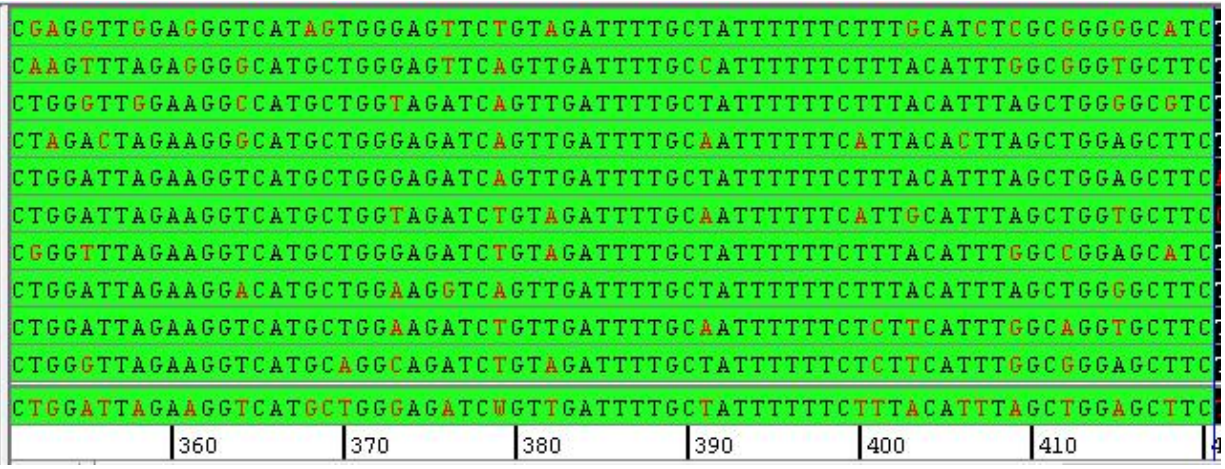

421-490 Nucleotide sequence alignment

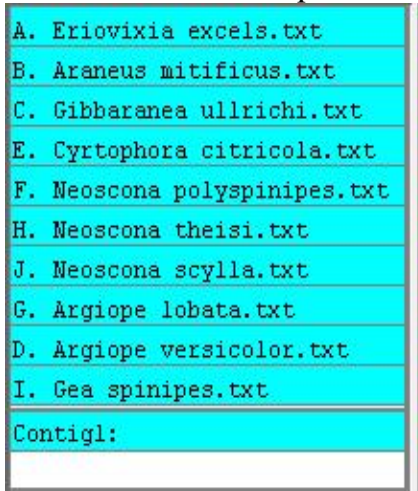

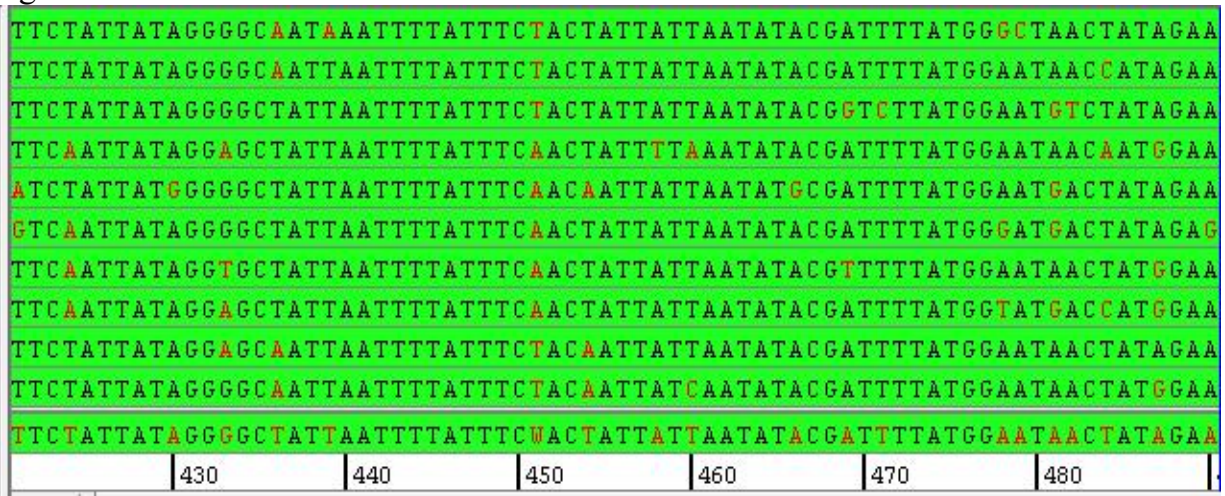

491-560 Nucleotide sequence alignment

\begin{tabular}{|c|c|}
\hline A. Eriovixia excels.txt & GTTCCTTTATTTGTATGATCGGTTTTGATCACGGCTGTACTATTATTATTGTCATTGCCTGTCTTGE \\
\hline B. Araneus mitificus.txt & AA ÄTACCTTTATTTGTATGATCAGTTTTAATTACAGCAGTTTTACTTTTACTCTCTTTACCTGTATTAG \\
\hline C. Gibbaranea ullrichi. txt & AAGGTTCCTTTATTTGTTTGATCTGTGTTAATTACAGCAGTTTTATTATTACTTTCTTTACCGGTTTTAG \\
\hline E. Cyrtophora citricola. txt & ÄДAGTCCTTTATTTGTTTGGTCTGTTCTÄTTACÄGCTGTTCTTCTATTATTATCTTTACCCGTTTTAG \\
\hline F. Neoscona polyspinipes. txt & A AGGTTCCTTTATTTGTATGATCAGTTTTGATTACTGCTGTTTTATTATTATTATCATTACCTGTATTAG \\
\hline H. Neoscona theisi. txt & Å̈GTACCTTTATTTGTÄTGATCTGTÄTTAATTACTGCÄGTÄTTATTATTATTATCTTTACCÄGTATTAG \\
\hline J. Neoscona scylla. txt & AAGGTACCTTTATTTGTCTGGTCAGTATTAATTACTGCAGTTTTATTATTGTTATCTTTACCTGTATTAG \\
\hline G. Argiope lobata. txt & AAGGTTCCTTTATTTGTTTGGTCTGTCTTAATTACAGCTGTACTTTTACTTTTATCATTGCCTGTATTAG \\
\hline D. Argiope versicolor. txt & Ä GGTTCCTTTATTTGTTTGATCAGTCTTAATTACTGCTGTTCTTTTACTTCTCTCGTTACCGGTTTTAC \\
\hline I. Gea spinipes. txt & AAGGTTCCTTTATTTGTTTGGTCTGTTCTAATTACTGCTGTTCTTCTTCTTTTGTCTTTACCTGTATTAG \\
\hline \multirow[t]{2}{*}{ Contigl: } & ÄAGGTTCCTTTATTTGTTTGATCTGTTTTAATTACTGCTGTTYTATTATTATTATCTTTACCTGTATTÁC \\
\hline & 520 \\
\hline
\end{tabular}

561-610 Nucleotide sequence alignment 

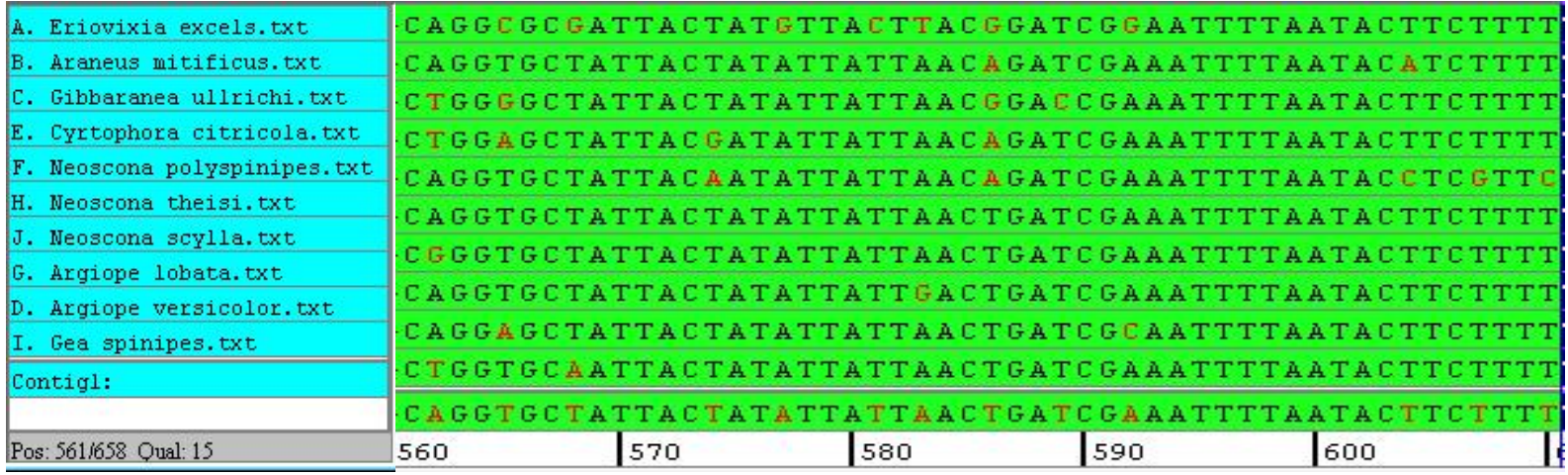

611-658 Nucleotide sequence alignment

\begin{tabular}{|l|}
\hline A. Eriovixia excels.txt \\
\hline B. Araneus mitificus.txt \\
\hline C. Gibbaranea ullrichi.txt \\
\hline E. Cyrtophora citricola.txt \\
\hline F. Neoscona polyspinipes.txt \\
\hline H. Neoscona theisi.txt \\
\hline J. Neoscona scylla.txt \\
\hline G. Argiope lobata.txt \\
\hline D. Argiope versicolor.txt \\
\hline I. Gea spinipes.txt \\
\hline Contigl: \\
\hline
\end{tabular}

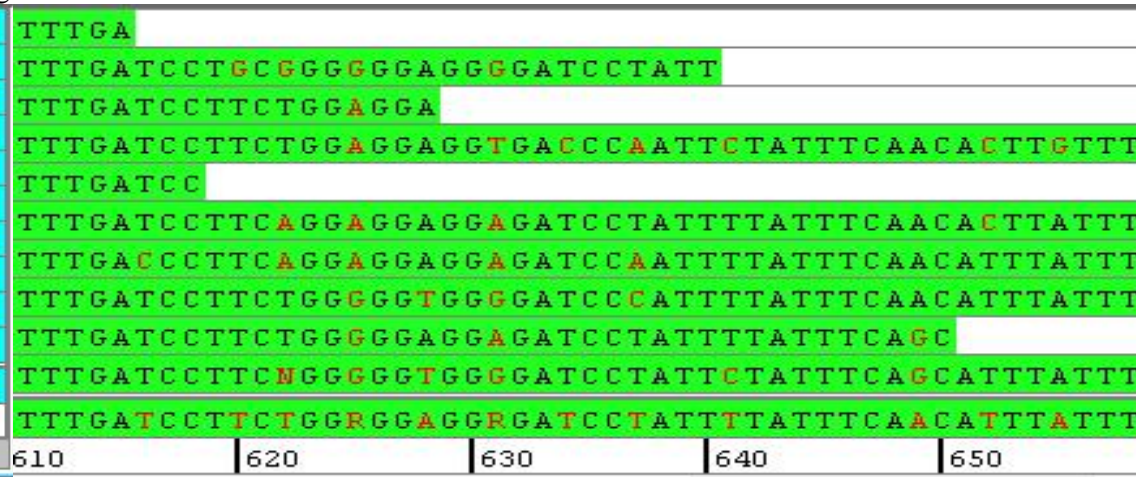

Figure 1: Nucleotide sequence alignments result of COI-5P generated by codon code aligner version 8.02 of the Eriovixia excels (Simon, 1889), Araneus mitificus (Simon, 1886) Gibbaranea ullrichi (Hahn, 1835), Argiope versicolor (Doleschall, 1859), Cyrtophora citricola (Forsskal, 1775), Neoscona polyspinipes (Yin, Wang, Xie \& Peng, 1990), Argiope lobata (Pallas, 1772), Neoscona theisi (Walckenaer, 1841), Gea spinipes (C. L. Koch, 1843) and Neoscona scylla (Karsch, 1879).

Gea spinipes(C. L. Koch, 1843)

Figs 3, 4, Map

Gea spinipes C. L. Koch, 1843: 101, figs 823 (D ㅇ). Gea spinipes Yin et al., 1997d: 90, figs 21a-f (ð゚+ ); Gea spinipes Chakrabarti, 2009: 128, figs 1-7 (ㅇ).

Sample id: ICUP-MS-0064, ICUP-MS-0087 and ICUPMS-0088

Process id: SPKPK064-20, SPKPK087-20 and SPKPK088-20

Match Process id: GACS1650-19

Material Examined: PAKISTAN: $1 \delta^{\Uparrow} 2 q$ (MICU, G-32), Goro stream, Talash, Dir Lower, Khyber Pakhtunkhwa $\left(34.44707^{\circ} \mathrm{N}, 71.49292^{\circ} \mathrm{E}\right), 768 \mathrm{~m}$ a.s.1., 09.07.2018, M. Sajid.

Distribution: The species was earlier known from India, China, Taiwan to Indonesia (WSC, 2020). The species is reported for the $1^{\text {st }}$ time in Northern Pakistan.

\section{Gibbaranea ullrichi (Hahn, 1835)}

Fig. 5, Map

Epeira ullrichii Hahn, 1835: 66, figs 159 (D $\overbrace{}^{\top}$ ) $).$

Araneus ullrichi Simon, 1929: 699, 765, figs 1091 (ð゚ㅇ); Gibbaranea ullrichi Heimer \& Nentwig, 1991: 82, figs $186\left({ }^{\uparrow}+\right.$ ) $)$.

Sample id: ICUP-MS-0022
Process id: SPKPK022-20

Match BIN: BOLD: AAD6909

Material Examined: PAKISTAN: 1 \& (MICU, 469), Redar Hills, Talash, Dir Lower, Khyber Pakhtunkhwa $\left(34.77247^{\circ} \mathrm{N}, 71.96441^{\circ} \mathrm{E}\right), 1868 \mathrm{~m}$ a.s.l., 21.09.2018, M. Sajid.

Distribution: This species has distribution from Europe to Central Asia (WSC, 2020). Northern areas of Pakistan are connected to Central Asian countries and the species has distribution pattern in Pakistan. There is no record of the species from India, Iran and Afghanistan the neighboring countries.

Neoscona polyspinipes Yin, Wang, Xie \& Peng, 1990 Fig 6, Map

Neoscona polyspinipes Yin et al., 1990: 104, figs 254261 (Dへ̂우).

Sample id: ICUP-MS-0042, ICUP-MS-0044, ICUPMS-0082 and ICUP-MS-0083

Process id: SPKPK042-20, SPKPK044-20, SPKPK08220 and SPKPK083-20

Match BIN: BOLD: AAO1983

Match Process id: AMSPK140-15

Material Examined: PAKISTAN: 2 (MICU, MP-77), Barcharay Hills, Talash, Dir Lower, Khyber Pakhtunkhwa $\left(34.71637^{\circ} \mathrm{N}, 71.91691^{\circ} \mathrm{E}\right), 1198 \mathrm{~m}$ a.s.1., $18.06 .2018 ; 2$ 우 
(MICU, S-63a,b), NagriPayeen, Talash, Dir Lower, Khyber Pakhtunkhwa $\left(34.74637^{\circ} \mathrm{N}, 71.93991^{\circ} \mathrm{E}\right), 1001 \mathrm{~m}$ a.s.1., 13.06.2018, all. coll. M. Sajid.

Distribution: The species was endemic to China (WSC, 2020). The present record from Pakistan represents the westernmost limit of the species distribution.

Neoscona scylla (Karsch, 1879)

Fig 7, Map

Epeira scylla Karsch, 1879g: 71 (D 9 ); Neoscona scylla Yaginuma, 1960: 56, fig 52.1(ぷ+); Neoscona scylla Baba \&Tanikawa, 2015: 66, fig 7 ( ఏ̊ + ).

Sample id: ICUP-MS-0089, ICUP-MS-0090, ICUP-MS0091, ICUP-MS-0092, ICUP-MS-0093 and ICUP-MS0094

Process id: SPKPK089-20, SPKPK090-20, SPKPK09120, SPKPK092-20, SPKPK093-20 and SPKPK094-20

Match BIN: BOLD: AAO1997

Match Process id: MASPD004-10

Material Examined: PAKISTAN: $2 q$ (MICU, SW-01, 02), NagriPayeen, Talash, Dir Lower, Khyber Pakhtunkhwa $\left(34.74767^{\circ} \mathrm{N}, 71.94041^{\circ} \mathrm{E}\right), 1010 \mathrm{~m}$ a.s.1., 14.08.2018, M. Sajid.

Distribution:The species is known from Russia (Far East), China, Korea and Japan (WSC, 2020). Present record from Northern areas of Pakistan represents the westernmost distributional limit of the species. This species is not recorded yet from adjacent India, Afghanistan and Iran but is likely to occur.

Neoscona theisi (Walckenaer, 1841)

Fig 8, Map

Epeira theisi Walckenaer, 1841: 53, pl. 18, f. 4 (Do).

Neoscona theisi Butt \& Siraj, 2006: 215, figs 1A-G (ð̊우).

Sample id: ICUP-MS-0061

Process id: SPKPK061-20

Match BIN: BOLD:ACM3489

Match Process id: GBMIN114644-17

Material Examined: PAKISTAN: 1 (MICU, MP-276), Pato Hills, Talash, Dir Lower, Khyber Pakhtunkhwa $\left(34.76277^{\circ} \mathrm{N}, 71.92491^{\circ} \mathrm{E}\right), 1238 \mathrm{~m}$ a.s.1., 21.07.2018, coll. M. Sajid.

Distribution: This species has a wide range of distribution from Cyprus, Israel, Russia, Iran, Pakistan, India, Philippines, China, Indonesia, Japan to Australia (WSC, 2020). The present record in Pakistan is the northernmost record in the country.

\section{Araneus mitificus (Simon, 1886)}

Figs 9, 10, Map

Epeira mitifica Simon, 1886a: $150 \quad$ (D+). Zilla nawazi Dyal, 1935:186, figs 6, pl. 16, fig 124 (Do); Araneus mitifica Biswas \& Raychaudhuri, 2013a: 158, figs 1-7 (ぷㅇ).

Sample id: ICUP-MS-0018 and ICUP-MS-0038
Process id: SPKPK018-20 and SPKPK038-20

Match BIN: BOLD: AAV1598

Match Process id: MTSPD326-15

Material Examined: PAKISTAN: 1 ㅇ 1 त (MICU, 804, 805), Kamranay Hills, Talash, Dir Lower, Khyber Pakhtunkhwa $\left(34.45974^{\circ} \mathrm{N}, 71.49475^{\circ} \mathrm{E}\right), 885 \mathrm{~m}$ a.s.1., 07.04.2019, M. Sajid.

Distribution: The species is distributed from India to Philippines, Japan, New Guinea (WSC, 2020). It was recorded from Eastern Pakistan (Dyal, 1935) and our record provides additional distributional data in the country.

\section{Eriovixia excelsa (Simon, 1889)}

Figs 12, Map

Glyptogona excelsa Simon, 1889i: 337 (Dㅇ).

Araneus excelsus Dyal, 1935: 179, pl. 16, figs. 116 (ㅇ)

Eriovixia excelsa Tso \&Tanikawa, 2000: 129, figs 17-22

( $\overbrace{}^{\lambda}+$ )

Eriovixia excelsa Caleb, 2020b: 15717, figs 3C, 24K (q).

Sample id: ICUP-MS-0005

Process id: SPKPK005-20

Match BIN: BOLD: AAQ0105

Match Process id: GBMIN114597-17

Material Examined: PAKISTAN: $2 q$ (MICU, 107, Fn09), Goro Hills, Dir Lower, Khyber Pakhtunkhwa $\left(34.44761^{\circ} \mathrm{N}, 71.49451^{\circ} \mathrm{E}\right), 818 \mathrm{~m}$ a.s.1., 02.09.2018, M. Sajid.

Distribution: This species has a wide distribution range from Pakistan, India, China, Taiwan, Philippines to Indonesia (WSC, 2020). It was previously recorded from eastern part of Pakistan (Lahore) (Dayal, 1935).The present record is the northernmost in the country.

\section{Cyrtophoracitricola (Forsskål, 1775)}

Figs 11, Map

Araneacitricola Forsskål, 1775: 86 (D).

Cyrtophoracitricola Dyal, 1935: 175, figs 111-115 (ㅇ);

Cyrtophoracitricola Nentwiget al., 2019: 35.

Sample id: ICUP-MS-0003 and ICUP-MS-0043

Process id: SPKPK003-20 and SPKPK043-20

Match BIN: BOLD: AAO2032

Match Process id: MASPD022-10

Material Examined: PAKISTAN: $5 q$ (MICU, MP-78a,b; MP-79 a,b,c), Barcharay Hills, Talash, Dir Lower, Khyber Pakhtunkhwa $\left(34.71661^{\circ} \mathrm{N}, 71.91693^{\circ} \mathrm{E}\right), 1290 \mathrm{~m}$ a.s.l., 18.06.2018, M. Sajid;4우 (MICU, MP-134 a,b; MP-148 a, b), Zombaqy Hills, Talash, Dir Lower, Khyber Pakhtunkhwa $\left(34.73717^{\circ} \mathrm{N}, 71.94790^{\circ} \mathrm{E}\right), 1028 \mathrm{~m}$ a.s.l., 01.07.2018, M. Sajid; 2 (MICU, MP-308), Pato Hills, Talash, Dir Lower, Khyber Pakhtunkhwa $\left(34.76297^{\circ} \mathrm{N}\right.$, 71.92481 $\left.{ }^{\circ} \mathrm{E}\right), 1248 \mathrm{~m}$ a.s.1., 21.07.2018, all. coll. M. Sajid. 
Distribution: This species is known from Southern Europe, Africa, Middle East, Pakistan, India, China, Japan, Introduced to Dominican Rep., Costa Rica, Colombia and Brazil (WSC, 2020). The species is recorded for the first time from the study area.

\section{Cyclosahexatuberculata Tikader, 1982}

Figs 13, 14, Map

Cyclosahexatuberculata Tikader, 1982a: 197, figs 382387 (Dへ̃o).

Material Examined: PAKISTAN: $1 \hat{\delta}, 19$ (MICU, 819, Fn-820), Goro Hills, Dir Lower, Khyber Pakhtunkhwa $\left(34.44761^{\circ} \mathrm{N}, 71.49451^{\circ} \mathrm{E}\right), 818 \mathrm{~m}$ a.s.l., 02.09.2018, M. Sajid.

Distribution: The species is distributed in Pakistan and India (WSC, 2020). It is recorded for the firsttimefrom the northern regions in Pakistan which is also the westernmost distribution limit of the species.

\section{DISCUSSION}

A total 35araneid specimens were collected from Dir Lower Pakistan out of which 8 genera and 11 species were identified. Of which 23 were sent for DNA barcode to the Centre for Biodiversity Genomics, Biodiversity Institute of Ontario, University of Guelph, Canada. All the eleven species were recorded for the first time from study area (Dir Lower), while six species, Argiope versicolor, Argiope lobata Pallas, 1772,Neoscona polyspinipes Yin, Wang, Xie\& Peng, 1990,Neoscona scyllaKarsch, 1879,Gea spinipes C. L. Koch, 1843and Gibbaraneaullrichi Hahn, 1835 are new to the araneid fauna of Pakistan.

Table 1: Checklist of family Araneidae from Pakistan.

\begin{tabular}{lll}
\hline 1 & Araneus alboquadratus & Dyal, 1935 \\
2 & Araneus altitudinum & Caporiacco, 1934a \\
3 & Araneus camilla & Dyal, 1935 \\
4 & Araneus formosus & Dyal, 1935 \\
5 & Araneus fulvus & Dyal, 1935 \\
6 & Araneus obscurissimus & Caporiacco, 1934 \\
7 & Araneus pontii & Caporiacco, 1934 \\
8 & Chorizopes & Mukhtar andTahir, \\
& rajanpurensis & C013 \\
9 & Cyclosa & Mukhtar \\
& chichawatniensis & andMushtaq, 2005 \\
10 & Cyclosa kashmirica & Caporiacco, 1934 \\
11 & Cyclosa krusa & Barrion and \\
& & Litsinger, 1995 \\
12 & Cyclosa mohini & Dyal, 1935 \\
13 & Cyclosa punjabiensis & Ghafoor\& Beg, 2002 \\
14 & Cyclosa saismarka & Barrion and Litsinger \\
& & 1995 \\
15 & Cyclosa spirifera & Simon, 1889 \\
\hline
\end{tabular}

\begin{tabular}{lll}
\hline 16 & $\begin{array}{l}\text { Cyrtarachne } \\
\text { nagasakiensis }\end{array}$ & Strand, 1918 \\
17 & Cyrtophora cicatrosa & Stoliczka, 1869 \\
18 & Cyrtophora citricola & Forsskål, 1775 \\
19 & Eriovixia excelsa & Simon, 1889 \\
20 & Gasteracantha dalyi & Pocock, 1900 \\
21 & Neoscona huzaifi & Mukhtar, 2012 \\
22 & Neoscona pavida & Simon, 1906 \\
23 & Neoscona theisi & Walckenaer, 1841, \\
24 & Nephila pakistaniensis & Ghafoor and Beg, \\
& & 2002 \\
25 & Araneus nympha & Simon, 1889 \\
26 & Argiope anasuja & Thorell, 1887 \\
27 & Cyclosa & Tikader, 1982 \\
& hexatuberculata, & \\
28 & Neoscona bengalensis & Tikader and Bal, \\
& & 1981 \\
29 & Argiope versicolor & Doleschall, 1859 \\
30 & Argiope lobate & Pallas, 1772 \\
31 & Neoscona polyspinipes & Yin, Wang, Xie and \\
& & Peng, 1990 \\
32 & Neoscona Scylla & Karsch, 1879 \\
33 & Gea spinipes & C. L. Koch, 1843 \\
34 & Gibbaranea ullrichi & Hahn, 1835 \\
\hline
\end{tabular}

The spider fauna of Pakistan is not yet fully explored due to lack of expertise and interest. Dyal [1935] reported, Cyrtophora citricola (Araneidae) from Lahore but did not record Neoscona theisi from the area which is very common now (Butt and Siraj, 2006). WSC (2020) lists only 32 species from Pakistan in which Araneus alboquadratus Dyal, 1935, Araneus altitudinum Caporiacco, 1934a, Araneus camilla Dyal, 1935, Araneus formosus Dyal, 1935, Araneus fulvus Dyal, 1935, Araneus obscurissimus Caporiacco,1934, Araneus pontii Caporiacco, 1934, Chorizopes rajanpurensis Mukhtar and Tahir, 2013,Cyclosa chichawatniensis Mukhtar and Mushtaq, 2005,Cyclosa kashmirica Caporiacco, 1934, Cyclosa krusa Barrion and Litsinger, 1995, Cyclosa mohini Dyal, 1935, Araneus nympha Simon, 1889, Argiope anasuja Thorell, 1887, Cyclosa hexatuberculata Tikader, 1982, Neoscona bengalensis Tikader and Bal, 1981, Cyclosa punjabiensis Ghafoor and Beg, 2002, Cyclosa saismarka Barrion and Litsinger 1995, Cyclosa spirifera Simon, 1889; Cyrtarachne nagasakiensis Strand, 1918, Cyrtophora cicatrosa Stoliczka, 1869, Cyrtophora citricola Forsskål, 1775; Eriovixia excels Simon, 1889,Gasteracantha dalyi Pocock, 1900, Neoscona huzaifi Mukhtar, 2012, Neoscona pavida Simon, 1906; Neoscona theisi Walckenaer, 1841, Nephila pakistaniensis Ghafoor and Beg, 2002 are confirmed from various localities of Pakistan. While Neoscona chrysanthusi Tikader and Bal, 1981, Neoscona mukerjei Tikader, 1980, Neoscona shillongensis Tikader and Bal, 1981 and Neoscona sinhagadensis Tikader, 1975 
have a doubtful record from the country because most of these species were recorded from India and there is no data about Pakistan.

With the addition of present data, the araneid list of Pakistan reaches to 38 species. Existence of 34 species were confirmed from the published literature including the new records of the present study while 4 species cited above has no evidence of existence in the country from the published literature at WSC. Further study and exploration efforts are necessary for documentation of the entire araneid fauna of Pakistan. There are great possibilities for the presence of many undescribed species. Moreover, species described from neighboring countries like China and India whose fauna are relatively well explored are likely to be present in Pakistan as well. An updated checklist is attached.

\section{REFERENCES}

Arshad, M., G.A. Jan and M. Iqbal (1984). Some spiders of Peshawar and adjoining areas. Zool. Sur. Pakistan, 10: 83-89.

Ashfaq, M., G. Blagoev, H.M. Tahir, A.M. Khan, M.K. Mukhtar, S. Akhtar, B. Abida, M. Shahid and D.N.H. Hebert (2019). Assembling a DNA barcode reference library for the spiders (Arachnida: Araneae) of Pakistan. PLoS ONE. 14(5): e0217086.

Baba, Y.G. and A. Tanikawa (2015). The handbook of spiders. Bun-ichi Sogo Shuppan, Tokyo. 112 p

Barrion, A.T. and J.A. Litsinger (1995). Riceland spiders of South and Southeast Asia. CAB International Wallingford, UK. P.700 p

Biswas, V. and D. Raychaudhuri (2013). Orb-weaving spiders of Bangladesh-I: genus Araneus Clerck (Araneae: Araneidae) with two new species. Records of the Zoological Survey of India. 113(2): 157-162.

Butt, A. and A. Siraj (2006). Some Orb Weaver Spiders from Punjab, Pakistan. Pakistan J. Zool. 38(3): 215-220.

Caleb, J.T.D. (2020). Spider (Arachnida: Araneae) fauna of the scrub jungle in the Madras Christian College campus, Chennai, India. J. Thre. Tax. 12(7): 15711-15766.

Caporiacco, L.D. (1934a). Aracnidi dell'Himalaia e del Karakoram raccolti dalla Mission eItaliana al Karakoram (1929-VII). Memorie della Società Entomologica Italiana. 13: 113-160.

Chakrabarti, S. (2009). Geaspinipes C. L. Koch 1843 (Araneae: Araneidae) found in western Himalaya, India. Turkish J. Arach. 1(2): 128-132.

Clerck, C. (1757). Svenska spindlar, uti sina hufvudslågter indelte samt under några och sextio särskildte arter beskrefne och med illuminerade figurer uplyste. Stockholmiae. P.154.
Doleschall, L. (1859). Tweede Bijdrage tot de kennis der Arachniden van den Indischen Archipel. Acta Societatis Scientiarum Indica-Neerlandica. 5: 160.

Dyal, S. (1935). Fauna of Lahore. 4.-Spiders of Lahore. Bulletin of the Department of Zoology of the Panjab University. 1: 119-252.

Forsskal, P. (1775). Descriptiones animalium avium, amphibiorum, piscium, insectorum, vermium; quae in itinere orientali observavit Petrus Forskål[sic]. Hauniae. 85-86 p

Gajbe, U.A. (2005). Studies on some spiders of the family Araneidae (Araneae: Arachnida) from Madhya Pradesh, Iindia. Record zoological Survey India. 105: 45-60.

Ghafoor, A. (2002). Taxonomic and some ecological studies of the cursorial spiders of cotton fields at Faisalabad, Pakistan. Ph.D. thesis, Deptt. of Zool and Fish, Univ. of Agri., Faisalabad, Pakistan.

Ghafoor, A. and M.A. Beg (2002). Description of two new species of araneid spiders from Pakistan. Inter. J. Agr. Bio. 4: 525-527.

Hahn, C.W. (1835). C. H. Zeh'sche Buchhandlung, Nürnberg, Zweiter Band. Die Arachniden. 57-75 $\mathrm{p}$

Heimer, S. and W. Nentwig (1991). Spinnen Mitteleuropas: EinBestimmungsbuch. Paul Parey, Berlin. 543 p

Jäger, P. (2012). A review on the spider genus Argiope Audouin 1826 with special emphasis on broken emboli in female epigynes (Araneae: Araneidae: Argiopinae). Beiträgezur Araneologie. 7: 272-331, 358-362.

Jäger, P. and B. Praxaysombath (2009). Spiders from Laos: new species and new records (Arachnida: Araneae). Acta Arach. 58: 27-51.

Karsch, F. (1879). Baustoffe zu einer Spinnenfauna von Japan. Verhandlungen des Naturhistorischen Vereins der Preussischen Rheinlande und Westfalens. 36: 57-105.

Kazim, M., P. Rukhsana, Z. Abid, H. Rafiq, F. Nadia and A. Sherzad (2015). Biodiversity of spider (Arachnida: Araneae) fauna of Gilgit Baltistan Pakistan. Int. J. Fauna and Biol. Stud. 2(4): $77-$ 79.

Khatoon, S. (1985-86). A checklist of Arachnids of Pakistan. Bull. Hydrobiol. Res. Kar. Pakistan 1: 645-6650.

Koch, C.L. (1843). C. H. Zeh'sche Buchhandlung, Nürnberg, Zehnter Band. Die Arachniden. 37$142 \mathrm{p}$

Mukhtar, M.K. and S. Mushtaq (2005). Spiders of the genus Cyclosa (Araneae: Araneidae) from Punjab, Pakistan. Pakistan J. Zool. 37: 199-204.

Mukhtar, M.K. (2012). Spiders of the genus Neoscona (Araneae: Araneidae) from 
Punjab, Pakistan. Pakistan J. Zool. 44: 17111720 .

Mukhtar, M.K. and H.M Tahir (2013). A new species of the genus Chorizopes (Araneae: Araneidae) from Punjab, Pakistan. Pakistan J. Zool. 45: 433-436.

Nentwig, W., T. Blick, D. Gloor, P. Jäger, and C. Kropf (2019). Tackling taxonomic redundancy in spiders: the infraspecific spider taxa described by Embrik Strand (Arachnida: Araneae). ArachnologischeMitteilungen. 58: 29-51.

Pallas, P.S. (1772). Spicilegia zoologica. Tomus 1. Continen squadrupedium, avium, amphibiorum, piscium, insectorum, molluscorum aliorumque marinorum fasciculos decem. Berolini. 1(9): 4450.

Parveen, R. (2003). Taxonomic study on some spider of Punjab, Pakistan. Thesis, Department of Zoology and Fisheries, Agriculture University Faisalabad, Pakistan. 1-261 p

Pocock, R.I. (1900). The fauna of British India, including Ceylon and Burma. Arachnida. Taylor and Francis, London. 279 p

Qadir, A. (1997). Taxonomic studies of spider's families Araneidae, Oxyopidae, Clubionidae and Eresidae of Sialkot. M.Sc. thesis, Department of Zoology and Fisheries, University of Agriculture, Faisalabad, Pakistan.

Razzaq, A. (2002). Taxonomical studies on spider fauna of Kaghan Valley, Pakistan. M.Phil. thesis, Department of Zoology and Fisheries, University of Agriculture, Faisalabad, Pakistan.

Simon, E. (1886). Arachnids collected by MA Pavie (Deputy Head of the Postal Service in Cambodia) in the Kingdom of Siam, Cambodia and Cochinchina. Proceedings of the SociétéLinnéenne de Bordeaux. 40: 137-166.

Simon, E. (1889). Arachnides de l'Himalaya, recueillis par MM. Oldham et Wood-Mason, et faisantpartie des collections de l'Indian Museum. Première partie.J.The Asiatic Society of Bengal, part II (Natural science). 58: 334-344.

Simon, E. (1906). Arachnides recueillis par L. Fea sur la côteoccidentale d'Afrique. 1re partie. Annali del Museo Civico di StoriaNaturale di Genova. 43: 218-323.

Simon, E. (1929). Les arachnides de France. Synopsis générale et catalogue des espècesfrançaises de l'ordre des Araneae. Tome VI. 3e partie. Roret, Paris. 533-772 p

Strand, E. (1918). Zur Kenntnis japanischer Spinnen, I and II. Archiv für Naturgeschichte. 82: 73-113. P1. 12.

Stoliczka, F. (1869). Contribution towards the knowledge of Indian Arachnoidea. J. Asiatic Soc. Beng. part II (Natural science) 38: 201-251.
Thorell, T. (1873). Remarks on synonyms of European spiders. Part IV. C. J. Lundström, Uppsala. P.375-645.

Thorell, T. (1887). Viaggio di L. Fea in Birmania e regionivicine. II. Primo saggio sui ragnibirmani. Anna. del Mus. Civico di Stor. Nat. di Genova. 25: 5-417.

Tikader, B.K. (1982). Part 1. Family Araneidae (Argiopidae). Typical orb-weavers. In: The fauna of India. Spiders: Araneae. II. Zoological Survey of India, Calcutta.1-293p

Tikader, B.K. (1975). Some new species of spiders of the family Argiopidae from India. Proceedings of the Indian Academy of Science. 81: 145-149.

Tikader, B.K. (1980). Description of a new species of spider of the genus Neoscona (Family: Araneidae) from India and some observations on intraspecific colour variation. Proceedings of the Indian Academy of Science (Anim. Sci.). 89: 247-252.

Tikader, B.K. and A. Bal (1981). Studies on some orbweaving spiders of the genera Neoscona Simon and Araneus Clerck of the family Araneidae (=Argiopidae) from India. Records of the Zoological Survey of India, Occasional Paper. 24: 1-60.

Tso, I.M. and A. Tanikawa (2000). New records of five orb-web spiders of the genera Leucauge, Mesida, and Eriovixia (Araneae: Tetragnathidae and Araneidae) from Taiwan. Acta Arach. 49: 125131.

Tyagi, K., Kumar, V., Kundu, S., Pakrashi, A., Prasad, P., Caleb, J.T.D. and Chandra, K. (2019). Identification of Indian spiders through DNA barcoding: cryptic species and species complex. Scientific Reports. 9(14033): 1-13.

Walckenaer, C.A. (1841). Histoire naturelle des Insects. Aptères. Tome deuxième. Roret, Paris. P.549 P1.16-22.

WSC, (2020). World Spider Catalog. Version 21.0. Natural History Museum Bern, online at http://wsc.nmbe.ch, [accessed on 16th April 2020]. doi: $10.24436 / 2$.

Yaginuma, T. (1960). Spiders of Japan in colour.Hoikusha, Osaka.186p

Yin, C.M., J.F, Wang, L.P. Xie, and X.J. Peng (1990). New and newly recorded species of the spiders of family Araneidae from China (Arachnida, Araneae). In: Spiders in China: One Hundred New and Newly Recorded Species of the Families Araneidae and Agelenidae. Hunan Normal University Press. 1-171 p

Yin C.M., J.F. Wang, M.S. Zhu, L.P. Xie, X.J. Peng, and Y.H. Bao (1997). Fauna Sinica: Arachnida: Araneae: Araneidae. Science Press, Beijing. 460 p. 
